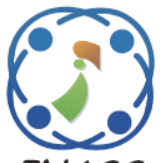

\title{
The Development of a Performance Appraisal System Using Decision Tree Analysis and Fuzzy Logic
}

\author{
Bryan Irvin Joven Lamarca ${ }^{1 *} \quad$ Shaneth Cueno Ambat ${ }^{2}$ \\ ${ }^{1}$ University of Northern Philippines, Philippines \\ ${ }^{2}$ AMA University, Philippines \\ * Corresponding author’s Email: bryanirvinlamarca@gmail.com
}

\begin{abstract}
The establishment of highly qualified faculty has become the core work of human resource management in State Universities and Colleges. Also, faculty add value to higher education institutions and performance evaluation is the best way to keep track of them. This study presents the development of a performance appraisal system which aims at studying the HR specific to the educational environment and brings out the role of data mining in achieving quality enhanced development in its faculty. The researchers utilized CRISP-DM and Extreme Programming methodologies, focusing on generating models for the Decision Tree algorithm, combined with Fuzzy Logic Controller in predicting faculty performance. J48-generated IF-THEN rules is utilized in conjunction with FLC to predict individual or institutional faculty performance. Also, the generated output of the prototype meets substantial standards. Finally, main users found the system to be Very Acceptable through IS0/IEC 20510:2011 software quality tool.
\end{abstract}

Keywords: CRISP-data mining, Decision tree, Fuzzy logic, Extreme programming, Performance appraisal.

\section{Introduction}

Most organizations use performance appraisal system to evaluate the efficiency and effectiveness of their employees. The performance of a faculty has been found to be dependent on a number of parameters broadly ranging from the individual's qualifications, experience, level of commitment, research activities undertaken to institutional support, financial feasibility, top management's support etc. These parameters act as performance indicators for an individual and group and subsequently can impact on the decision making of the individual and also the stakeholders. However, the difficulty of evaluating the action of people is related to diversity in the strategies of involvement; unsuitable conditions for analysis and understanding for those that conduct the evaluation; and the subjective nature of the object of evaluation. In most situations the evaluation of staff performance may be influenced by the appraiser's experience, sensitivity and standards. Therefore, the scores awarded by the appraiser are only approximations and there is an inherent vagueness in the evaluation. This is because, in many circumstances, appraiser tends to use vaguely defined qualitative criteria in evaluating the performance of their subordinates [1]. Fuzzy logic maybe used in the evaluation approach because the performance of the appraisal involves the measurement of ability, competence and skills, which are fuzzy concepts that may be captured in fuzzy terms. As a result, fuzzy logic approach can be implemented to manage the uncertainty of information and ensure quality involved in staff performance evaluation [2]. Also, researchers have proven the success of fuzzy set theory in solving multiple criteria problems [3].

Teachers' performance evaluation incentives as the basis for school reforms have recently attracted support among governing bodies in the Philippines. The Civil Service Commission (CSC) implemented the Strategic Performance Management System (SPMS) which focuses on measures of performance based in assessing organizational and collective 
individual performance within government organizations. The organizational and individual major final outputs and success indicators are aligned to facilitate cascading of organizational and individual performance ratings. The different heads of offices determine final assessment of performance level of the individual employees based on proof of performance through the Individual Performance Commitment Review (IPCR). Performance measures follow the following categories: Effectiveness/Quality, Efficiency, and Timeliness utilizing a five-point scale (1 to 5), 5 being the highest and 1, the lowest. The eligibility of faculty for the Performance Based Bonus (PBB) will be based on their IPCR ratings. With University of Northern Philippines being a State University, this performance evaluation scheme is observed and implemented.

Given Data Mining's prominent use in HR activities [4], it is considered as the most suitable technology in giving additional insight into educational entities such as; student, lecturer, staff, alumni and managerial behavior. It acts as an active automated assistant in helping them to make better decisions on their educational activities. The rationale behind using Data Mining in this study is to predict the quality, productivity, and potential of faculty across various disciplines which will enable higher level authorities to take decisions and understand certain patterns of teacher's motivation, satisfaction, growth, and decline. By knowing the expenses, by studying the investments on research, education, and administration, the strategic planning and the effective and efficient allocation of the financial power can be much better. Also, it is important that when administrators make decisions and provide feedback to teachers on their performance, that the information is a valid measure of their actual job performance, which means it should include a teachers' responsibilities both inclass and out-of-class.

The general objective of this study is to create a framework that will be used in the development of a performance appraisal prototype that will be used in a SUC setting. Specifically, it aims to: a) Identify possible predictors substantial to the prediction of performance appraisal of faculty; b) Utilize data mining algorithm to discover knowledge from the data gathered; c) Apply the data model generated from the data mining algorithm in the development of the performance appraisal system; d) Evaluate the reliability of the output of the data mining algorithm; and e) Evaluate the software quality of the performance appraisal system in terms of
ISO/IEC 25010:2011 software quality assessment tool.

\section{Methods}

The main data sets for data mining will be collected from the Human Resource Management Office, University Planning and Information System Management Office, and Office of the Vice President of Academic Affairs. The data consists of faculty personal information, education information, professional information, and performance information. CRISP-DM techniques like business understanding, data understanding, and data preparation will be used to process, clean, and consolidate faculty data. Rigorous literature review will be undertaken together with CRISP-DM to identify appropriate data mining algorithm and fuzzy logic library to be used in the development of the prototype. Next, the data set is analysed through WEKA to identify patterns that represent relationship among data by applying chosen DM. Feature selection through WEKA's InfoGainAttributeEval will be conducted to identify attributes that can be remove that may strengthen the prediction accuracy of the generated model. Then rule sets generated from the classification model will be entered into the developed prototype in IFTHEN rules form. The values and practices promoted by Extreme Programming (XP) methodology will be observed in developing the prototype for this study. In addition, the researchers utilized HTML, CSS, PHP, JavaScript and other plugins, WAMP, MySQL, and Java for the software development and in implementing fuzzy logic and decision tree. Cohen's Kappa will be used to measure the reliability of the generated output of the prototype. Finally, users will evaluate the software quality of the prototype via ISO/IEC 25010:2011 software quality assessment tool. Functionality, reliability, and usability were the focus the assessment tool due to [5] ease of evaluation by professionals in contrast to the remaining characteristics. Indicators from widely used software evaluation models [6-8] were also incorporated. The responses for the items will be measured by means of five-point Likert scale.

\section{Results}

Table 2. Ranked variables through information gain 


\begin{tabular}{cll}
\hline Rank & InfoGain & \multicolumn{1}{c}{ Attribute } \\
\hline 1 & 0.068294 & Rank \\
\hline 2 & 0.036638 & highest_educational_attainment \\
\hline 3 & 0.027916 & Exp \\
\hline 4 & 0.016643 & has_designation \\
\hline 5 & 0.011534 & has_research_engagement \\
\hline 6 & 0.009948 & Sex \\
\hline 7 & 0.009332 & Age \\
\hline 8 & 0.004052 & is_studentorg_adviser \\
\hline 9 & 0.003712 & has_cs_eligibility \\
\hline 10 & 0.00359 & civil_status \\
\hline 11 & 0.002849 & teaching_evaluation \\
\hline 12 & 0.00218 & with_children \\
\hline 13 & 0.001734 & has_extension_engagement \\
\hline 14 & 0.001294 & has_overload \\
\hline 15 & 0.000945 & has_other_designation \\
\hline 17 & 0.00093 & has_production_activity \\
\hline 18 & 0.000826 & address_outside_vigan \\
\hline
\end{tabular}

\subsection{On identifying possible predictors substantial to the prediction of performance appraisal of faculty}

Data preparation and selection techniques utilized through CRISP-DM led to 4138 regular faculty instances. Noting earlier studies such as [914], the following group of attributes has been selected to be tested against employee performance: a) Personal information such as: age, gender, marital status and number of kids (if any); b) Education information such as: university type, general specialization, degree and grade, (3) Professional information such as: number of experience years, job title, rank, service period, and salary. Information gain attribute evaluation was utilized in WEKA to further enhance hidden insights from the data sets. Table 2 reveals that faculty rank is the most important among the set of predictors in performance evaluation.

\subsection{On utilizing data mining algorithm to discover knowledge from the data gathered.}

Through rigorous literature review discussions, the researchers used decision tree since it is the most utilized DM technique in producing prediction models and in giving best results. The process involved Decision Tree Testing using Training sets and Decision Tree Data Model generation through WEKA. 10-fold cross-validation was utilized wherein the dataset is divided into 10 pieces, and then hold out each of these pieces in turn for testing, train on the rest, do the testing and average the 10

$$
\text { IF highest_educational_attainment }=\text { Masters }
$$

AND exp <= 15

AND has_production_activity $=$ no

AND rank = Instructor 1

AND is_studentorg_adviser $=$ no

AND age $<=27$

AND age $<=24$

THEN pbb is GOOD (14.0)

IF highest_educational_attainment $=$ Masters

AND exp $<=15$

AND has_production_activity $=$ no

AND rank = Instructor 1

AND is_studentorg_adviser $=$ no

AND age $<=27$

AND age $>24$

AND with_children $=$ yes

AND age $<=26$

THEN pbb is BETTER (20.0)

IF highest_educational_attainment $=$ Masters

AND $\exp <=15$

AND has_production_activity $=$ no

AND rank = Instructor 1

AND is_studentorg_adviser $=$ no

AND age $<=27$

AND age $>24$

AND with_children $=$ yes

AND age $>26$

AND address_outside_vigan $=$ yes

THEN pbb is BETTER (3.0)

Figure. 1 Extracted rule sets from $\mathrm{j} 48$

Table 3. J48 confusion matrix

\begin{tabular}{lcccc}
\hline \multicolumn{1}{c}{$\begin{array}{c}\text { Observed } \\
\text { Value }\end{array}$} & \multicolumn{3}{c}{ Predicted } & Accuracy \\
\cline { 2 - 4 } & Good & Better & Best & \\
\hline & & & & $84.97 \%$ \\
\hline Good & 2061 & 158 & 70 & \\
\hline Average & 192 & 971 & 49 & \\
Percentage & & & 474 & \\
\hline $\begin{array}{l}\text { Correctly Classified Instances: } \\
\text { Incorrectly Classified Instances: }\end{array}$ & 3516 & $84.9686 \%$ \\
\hline
\end{tabular}

results. Once the cross validation is done, Weka invokes the learning algorithm 11 times, once for each fold of the cross-validation and then a final time on the entire dataset to get an evaluation result and estimate of the error, and then finally get the model to be used in the prototype.

Highest educational attainment is taken as root node from which experience, rank as branch nodes and so on. The knowledge represented by decision tree is extracted and represented in the form of IFTHEN rules for easier translation, some are displayed in Fig. 1.

The confusion matrix (as seen in Table 3) was used in determining the accuracy of the model 
wherein accuracy results can be calculated with the number of correct classifications divided by the total number of classifications.

Evidently, the confusion matrix highlights the following:

- The decision tree has classified 2061 Good objects as Good, 158 as Better, and 70 as Best, leading to 228 misclassifications.

- The decision tree has classified 971 Better objects as Better, 192 as Good, and 49 as Best, leading to 241 misclassifications.

- The decision tree has classified 474 Best objects as Best, 110 as Good, and 53 as Better, leading to 163 misclassifications.

\subsection{On applying the data model generated from data mining algorithm in the development of the performance appraisal system}

The Performance Appraisal Prototype consists of two interacting systems, a Java-based desktop application and a web-based application. The webbased application is solely used for evaluating the individual faculty while the java-based desktop application utilizes fuzzy logic for determining performance output. The faculty performance evaluation model used in the Java Application was adapted from IPCR evaluation 2 core Major Final Outputs (MFOs) - Core and Support. Characteristics defined by the Core factor are: Designation, Instruction, Production, Research, and Extension.

The concept of fuzzy set and the membership functions is utilized both in the Web Application and Java Application to map the linguistic characteristics of faculty performance that are either ranked Very High, High, Medium, Low, or Very Low by the deans/heads of the academic units. The degree of membership in fuzzy set is $[1,0]$, where ' 1 ' represent highest membership and ' 0 ' represents no membership. The fuzzy variable set and their membership value is defined as shown in Table 4.

Both the web application and java application system components interact among each other to perform their functionalities in achieving the results.

Table 4. Fuzzy variables for input parameters for quantity, quality, and timeliness of each evaluation item

\begin{tabular}{cc}
\hline Fuzzy Variable & Degree of Membership \\
\hline Outstanding & 1.0 \\
\hline Very Satisfactory & 0.8 \\
\hline Satisfactory & 0.6 \\
\hline Unsatisfactory & 0.4 \\
\hline Poor & 0.2
\end{tabular}

Fuzzy Logic Controller (FLC) was utilized in determining the output of faculty performance.
Based on fuzzy logic, a FLC [15] is a software component that controls the output variables of a system according to its inputs and a set of rules expressed with the uncertainty of human terms. jfuzzylite, a free and open-source FLC library programmed in Java [16] was utilized due to costly licensing and restrictive limitations brought about by widely-known libraries such as Matlab Fuzzy Logic Toolbox, Octave Fuzzy Logic Toolkit, and jFuzzyLogic. The following options where used for the FLC Engine: a) Controller - Mamdani, b) Linguistic Term - Triangle, c) T-Norms - minimum, d) S-Norms - maximum, and e) Defuzzifier centroid.

Fig. 2 shows how each of the sub-items in an MFO for an employee is computed through the fuzzy logic engine. Each sub-item's linguistic characteristics are computed by multiplying the subitem's weight and the degree of membership assigned, after which all of the results of the subitems are computed for each MFO area. The same model is used in the computation of the total of all areas under the CORE MFO.

1: for each item $i$ in $\mathrm{S}$ do

2: Initialize fuzzy logic control engine

3: $\quad$ Set engine name

4: $\quad$ Initialize input variable

5: $\quad$ Set name for input variable

6: $\quad$ Set range minimum range to 0.000 and maximum range to itemweight for input variable

7: $\quad$ Add triangle linguistic term for VERYLOW $(0.000$, itemweight $* 0.10$, itemweight $* 0.20$ ) to input variable

8: $\quad$ Add triangle linguistic term for LOW (itemweight $* 0.10$, itemweight $* 0.20$, itemweight $* 0.40$ ) to input variable

9: $\quad$ Add triangle linguistic term for MEDIUM (itemweight $* 0.20$, itemweight $* 0.40$, itemweight $* 0.60)$ to input variable

10: $\quad$ Add triangle linguistic term for $\mathrm{HIGH}$ (itemweight $* 0.40$, itemweight $* 0.60$, itemweight $* .80)$ to input variable

11: Add triangle linguistic term for VERYHIGH (itemweight $* 0.60$, itemweight $*$ 0.80 , itemweight) to input variable

12: $\quad$ Add input variable to fuzzy logic control engine

13: $\quad$ Initialize output variable

14: $\quad$ Set name for output variable

15: $\quad$ Set range minimum range to 0.000 and maximum range to itemweight for output variable

16: Set default value for output

17: Add triangle linguistic term for POOR (0.000, itemweight $* 0.10$, itemweight $*$ 
0.20) to output variable

18: $\quad$ Add triangle linguistic term for

UNSATISFACTORY (itemweight $* 0.10$,

Itemweight $* 0.20$, itemweight $* 0.40$ ) to output variable

19: $\quad$ Add triangle linguistic term for

SATISFACTORY (itemweight $* 0.20$, itemweight * 0.40, itemweight * 0.60) to output variable

20: $\quad$ Add triangle linguistic term for

VERYSATISFACTORY (itemweight $* 0.40$, itemweight $* 0.60$, itemweight $* .80)$ to output variable

21: $\quad$ Add triangle linguistic term for

OUTSTANDING (itemweight $* 0.60$, itemweight

* 0.80, itemweight) to output variable

22: $\quad$ Add output variable to fuzzy logic control engine

23: Initialize rule block

24: $\quad$ Add "if input is VERYLOW then output is POOR" to rule block

25: Add "if input is LOW then output is

UNSATISFACTORY" to rule block

26: $\quad$ Add "if input is MEDIUM then output is

SATISFACTORY" to rule block

27: $\quad$ Add "if input is HIGH then output is

VERYSATISFACTORY" to rule block

28: Add "if input is VERYHIGH then output is

OUTSTANDING" to rule block

29: Add rule block to fuzzy logic control engine

30: $\quad$ Set T-norms to Minimum

31: $\quad$ Set S-Norms to Maximum

32: $\quad$ Set Deffuzifier to centroid

33: $\quad$ Set value for input

34: $\quad$ Run engine

35: Display fuzzy output

36: Instantiate new rule block to get engine rule block

37: for all Rule $r$ do

38: $\quad$ if activation degree based from rule block conjunction and disjunction is true then

39:

40: Display rule activated

41: $\quad$ end for

42: end for

Figure.2 Java fuzzy logic controller pseudo code for computing item weights

The inference rules to compute for item/area rating are the following:

- if Input is very low then Output is poor

- if Input is low then Output is unsatisfactory

- if Input is medium then Output is satisfactory

- if Input is high then Output is very satisfactory
- if Input is very high then Output is outstanding

Take Core Function as an example and utilizing the linguistic variables defined in Fig. 3, the inference rules to compute for IPCR Evaluation are the following:

- if Core_Function is low then IPCR Evaluation is good

- if Core_Function is medium then IPCR_Evaluation is better

- if Core_Function is high then IPCR_Evaluation is best

After completion computations of the CORE and Support MFOs, the Performance Appraisal System executes the code below (as shown in Fig. 4) to display the predicted faculty performance.
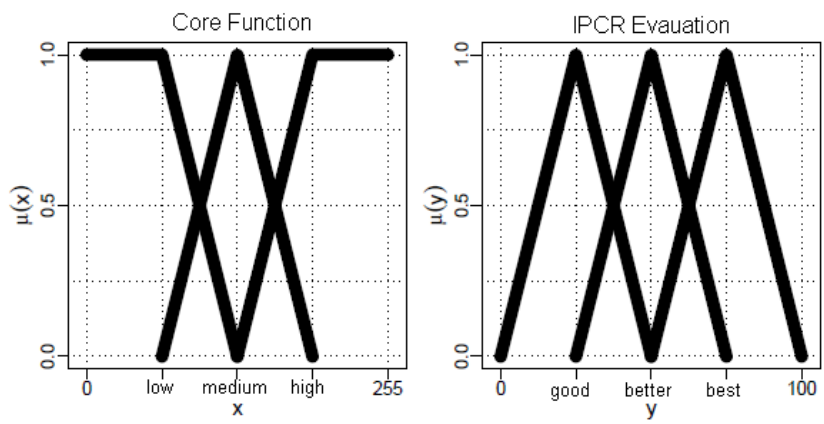

Figure. 3 Linguistic Variable Used in the Prototype

1: Initialize fuzzy logic control engine

2: Set engine name

3: Initialize input variable

4: Set name for input variable

5: Set range minimum range to 0.000 and maximum range to 1.000 for input variable

6: Add triangle linguistic term for $\operatorname{LOW}(0.000,0.250$, $0.500)$ to input variable

7: Add triangle linguistic term for MEDIUM (0.250, $0.500,0.750)$ to input variable

8: Add triangle linguistic term for $\mathrm{HIGH}(0.500$, $0.750,1.000)$ to input variable

9: Add input variable to fuzzy logic control engine

10: Initialize output variable

11: Set name for output variable

12: Set range minimum range to 0.000 and maximum range to 1.000 for output variable

13: Set default value for output

14: Add triangle linguistic term for GOOD (0.000, $0.250,0.500)$ to output variable

15: Add triangle linguistic term for BETTER $(0.250$, $0.500,0.750)$ to output variable

16: Add triangle linguistic term for BEST $(0.500$, $0.750,1.000)$ to output variable

17: Add output variable to fuzzy logic control engine 
18: Initialize rule block

19: Add "if input is LOW then output is GOOD" to rule block

20: Add "if input is MEDIUM then output is BETTER" to rule block

21: Add "if input is HIGH then output is BEST" to rule block

22: Add rule block to fuzz logic control engine

23: Set T-norms to Minimum

24: Set S-Norms to Maximum

25: Set Deffuzifier to centroid

26: Set value for input

27: Run engine

28: Display fuzzy output

29: Instantiate new rule block to get engine rule block

30: for all Rule $r$ do

31: $\quad$ if activation degree based from rule block conjunction and disjunction is true then

32: Display rule activated

33: $\quad$ end if

34: end for

Figure. 4 Java fuzzy logic controller pseudo code

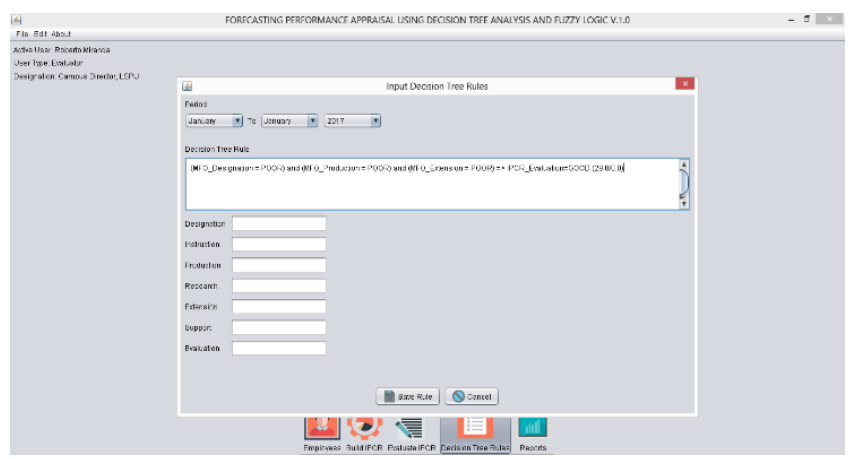

Figure. 5 Input decision tree rule set form module

The extracted rule sets from the model will be stored in the database through a form (as shown in Fig. 5) in the system that will be used in generating output. The Performance Appraisal prototype can produce output based on FLC or output from FLC mapped with the result sets. The evaluation output includes rating of Major Final Outputs, IPCR rating, and the rule sets satisfied by faculty variables. In addition, the system can generate predicted performance of each of the faculty under the colleges or generate institutional predicted performance of all faculty.

\subsection{On evaluating the reliability of the output of the data mining algorithm}

The reliability of this computational solution is complexly tied to the accuracy of the data used in the development of the prototype. Measuring the reliability of the generated output of the prototype is accomplished by comparing it to the decision rendered manually by the evaluators of each academic unit. For convenience, the calculations were done with SPSS. The Kappa computation yielded the values as presented in the Table 5, which translates the output of the prototype meets substantial standards.

\subsection{On evaluating the software quality of the performance appraisal system}

A questionnaire was distributed to president, vice presidents, deans and academic heads, and other personnel involved in implementation of SPMS and processing of PBB of employees asking them to rate the prototype's ability to display faculty performance based on the concept of FLC or FLC results mapped with result sets. The survey revealed that majority of the users found the system to be helpful in their task/job.

The results shown in Table 6 describe the interpretation of the respondents' evaluation in terms of functionality. According to the result, the system can perform the task required and able to produce the expected result with the overall average mean of 4.30 and interpreted as Very Good. Also, an overall average weight mean of 3.68 for usability was given with the interpretation of Very Good. In addition, the prototype is reliable with an average weighted mean of 4.02, Very Good. In summary, the prototype received an overall mean of 4.22 which means that the respondents view the prototype to be functional, usable, and reliable.

Table 5. Kappa results

\begin{tabular}{ccc}
\hline Evaluator & Kappa & Interpretation \\
\hline 1 & 0.333 & Fair \\
\hline 2 & .144 & Slight \\
\hline 3 & 0.84 & Almost Perfect \\
\hline 4 & 0.667 & Substantial \\
\hline 5 & 0.74 & Substantial \\
\hline 6 & 0.394 & Fair Agreement \\
\hline 7 & 0.87 & Almost Perfect \\
\hline 8 & 0.79 & Substantial \\
\hline 9 & 0.667 & Substantial \\
\hline 10 & 1.00 & Almost Perfect \\
\hline 11 & 0.53 & Moderate \\
\hline 12 & 0.75 & Substantial \\
\hline 13 & 0.615 & Substantial \\
\hline 14 & 0.500 & Moderate \\
\hline 15 & 0.65 & Substantial \\
\hline 16 & 0.74 & Substantial \\
\hline 17 & 0.61 & Substantial \\
\hline 18 & 0.66 & Substantial \\
\hline 19 & 0.92 & Almost Perfect \\
\hline
\end{tabular}


Table 6. Respondents' assessment of the prototype

\begin{tabular}{|c|c|c|}
\hline $\begin{array}{c}\text { Software } \\
\text { Characteristics }\end{array}$ & $\begin{array}{l}\text { Weighted } \\
\text { Mean }\end{array}$ & $\begin{array}{c}\text { Verbal } \\
\text { Interpretation }\end{array}$ \\
\hline \multicolumn{3}{|l|}{ FUNCTIONALITY } \\
\hline 1 & 4.45 & Agree \\
\hline 2 & 4.39 & Agree \\
\hline 3 & 4.48 & Excellent \\
\hline 4 & 4.30 & Agree \\
\hline 5 & 4.27 & Agree \\
\hline 6 & 4.30 & Agree \\
\hline Functionality Mean & 4.37 & Very Good \\
\hline \multicolumn{3}{|l|}{ USABILITY } \\
\hline 1 & 3.48 & Neutral \\
\hline 2 & 3.52 & Agree \\
\hline 3 & 3.48 & Neutral \\
\hline 4 & 4.39 & Agree \\
\hline 5 & 3.52 & Agree \\
\hline Usability Mean & 3.68 & Very Good \\
\hline \multicolumn{3}{|l|}{ RELIABILITY } \\
\hline 1 & 4.03 & Agree \\
\hline 2 & 3.97 & Agree \\
\hline 3 & 3.76 & Agree \\
\hline 4 & 4.30 & Agree \\
\hline Reliability Mean & 4.02 & Very Good \\
\hline Overall Mean & 4.14 & Very Good \\
\hline $\begin{array}{l}\text { Legend: } 4.51-5.00-\text { Exc } \\
2.51-3.50-\text { Good, } 1.51-2\end{array}$ & $\begin{array}{l}\text { ent, } 3.51-4 \\
\text { 0-Fair, and }\end{array}$ & $\begin{array}{l}\text {-Very Good, } \\
00-1.50 \text {-Poor }\end{array}$ \\
\hline
\end{tabular}

\section{Discussion}

Rigorous literature review together with CRIPSM_DM processes and techniques paved the way to the identification of data sets and setting data mining goals resulting to 3516 instances of faculty information collected from different offices concerned. 18 predictors were identified from pertinent information of regular faculty. Also, information gain was also utilized to shed light to variables that can be removed from the data sets that can possibly increase the prediction accuracy of the data mining algorithm. However, trial and testing by removing combination of attributes with lesser information gain only lessen the prediction accuracy of the model. Hence, the researchers just decided to push through with the default J48 classification results generated from WEKA as discussed below.

The J48 classification results generated from WEKA ten-fold cross validation yielded 3516 correctly classified instances out of 4138 data sets resulting to $84.9686 \%$ accuracy. Data mining experts were consulted to ensure the researchers will achieve data mining goals and objectives. The researchers initially started with 304 regular faculty based on training sets and the accuracy of the prediction model was just $54.37 \%$. The consulted experts suggested to collect more substantial data, hence regular faculty data from S.Y. 2012-2016 were collected and produced the results presented in the study and producing more significant result sets for predicting faculty performance in the process.

The Performance Appraisal Prototype consists of a Java-based desktop application and a web-based application. The web-based application utilized fuzzy sets and membership function to map qualitative variables as numeric ones based from the performance measures based from IPCR from SPMS, a performance scheme developed by the CSC. Also, the web-based application is solely used for evaluating the individual faculty while the javabased desktop application utilized Fuzzy Logic Controller (FLC) for determining performance output. Extracted rule sets from the J48 classification model in IF-THEN rules format can be stored in the database through the Decision Tree Input Rule Set Module in the Java Desktop Application component that can be used in generating faculty performance appraisal output. The Performance Appraisal prototype is able to produce output based on FLC or output from FLC mapped with the result sets. The evaluation output includes rating of Major Final Outputs, IPCR rating, and the rule sets satisfied by faculty variables. Also, the prototype can predict performances of faculty under the colleges or predict institutional performance of all faculty. To support decision making, the module for predicting faculty performance provides bases for reports such as consistent performers, regressing performers, and improved performers. Warnings are given to the supervisor towards regressing faculty to yield proper action. The Decision Tree Input Rule Set Module is one of the highlights of the prototype because it rids users from hiring programmers to incorporate/hard code generated rule sets in the module. Data analysts of the university will just have to input rule sets into the system. Furthermore, the Decision Tree Input Rule Set Module accepts results generated from other classification models generated from WEKA.

Cohen's Kappa was used to measure the reliability of the generated output of the prototype was accomplished by comparing it to the decision rendered manually by the evaluators of each academic unit head. The Kappa computation yielded the values which translates the output of the prototype meets substantial standards coinciding with the decent J48 classification accuracy.

The overall acceptability of the Performance Appraisal System based on the perception of deans/academic heads, executive officials, and officials overseeing the SPMS resulted to an overall mean of 4.14 which has an interpretation of Very 
Good. This translates that the prototype is functional, reliable, and usable. The evaluation process was time consuming because there is a need to explain the concepts behind the prototype. The evaluators appreciate a fresh take on evaluating faculty performance. However, respondents observed that the computation of performance appraisal was a little bit slow. Ref. [16] argued that Mamdani controllers are more computationally expensive because defuzzifiers generally need to integrate over the resulting fuzzy sets, whereas defuzzifiers in Takagi-Sugeno would consist only of a few arithmetic operations. Also, a more meaningful result of the prototype acceptability would have been elicited if the faculty was included as respondents.

\section{Conclusions}

The researchers were able to fulfill all the objectives of the study, therefore affirming the following conclusions:

1. CRISP-DM techniques with the aid of rigorous literature review of significant concepts led to the determined predictors for predicting faculty performance appraisal.

2. A J48 classification model was generated from WEKA for predicting faculty performance appraisal.

3. The rule sets for predicting Good, Better, and Best faculty performance can be stored in the database and utilized in conjunction with Fuzzy Logic Controller in the Performance Appraisal System that produce evaluation output with information vital for decision making. Also, the prototype can predict individual or institutional performances of faculty.

4. The reliability of the generated output of the prototype meets substantial standards.

5. The prototype is found to be very functional, usable, and reliable.

\section{Recommendations}

The domain of the study is still in its infancy and there is a vast space to explore. The following are recommendations future researchers may want to dwell into:

1. The application of the combination and fuzzy logic and decision tree in other domains aside from Human Resources.

2. Utilization of data sets including all types of employees in the SUC.

3. The generation and testing of other models with the use of other classification techniques other than decision tree to be used as input in the prototype.

4. Application of other Fuzzy Controllers other than Mandani to improve computation performance.

5. Testing and feasibility study of the prototype by other government organizations to aim for national scope implementation.

\section{References}

[1] G. Meenakshi, "Multi source feedback based performance appraisal system using Fuzzy logic decision support system", arXiv preprint arXiv:1203.1882., 2012.

[2] A. Neogi, A.C. Mondal, and S.K. Mandal, "A cascaded fuzzy inference system for university non-teaching staff performance appraisal", Journal of Information Processing Systems, Vol. 7, No. 4, 595-612, 2011.

[3] A.A. Yusof, "Performance Appraisal: Issues, Challenges \& Prospects. Prentice Hall”, 2003.

[4] H. Jantan, A. R. Hamdan, and Z. U. Othman, "Knowledge Discovery Techniques for Talent Forecasting in Human Resource Application", World Academy of Science, Engineering and Technology, International Journal of Social, Behavioral, Educational, Economic, Business and Industrial Engineering, Vol.3, No.2, pp. 178-186, 2009

[5] B.B. Chua and L.E. Dyson, "Applying the ISO 9126 model to the evaluation of an e-learning system", In: Proc. of ASCILITE, pp. 5-8, 2004.

[6] F.D. Davis, Perceived usefulness, perceived ease of use, and user acceptance of information technology, MIS quarterly, pp. 319-340, 1989.

[7] T.S. Tullis and J.N. Stetson, "A comparison of questionnaires for assessing website usability", In: Proc. of Usability professional association conference, pp. 1-12, 2004.

[8] I. Padayachee, P. Kotze, and A. van Der Merwe, ISO 9126 external systems quality characteristics, sub-characteristics and domain specific criteria for evaluating e Learning systems, The Southern African Computer Lecturers' Association, University of Pretoria, South Africa, 2010.

[9] H. Jantan, A. R. Hamdan, and Z.A. Othman, Data Mining Classification Techniques for Human Talent Forecasting. KnowledgeOriented Applications in Data Mining, InTech, 2011.

[10] A. Fatima and S. Rahaman, "Mining System in HR: A Proposed Model", International Journal 
of Computer and Information Technology, Vol. 3, No. 5, 2014.

[11] Mohammad and H. Alhaidey, "Implementation of a Training Courses Recommender System based on k-means algorithm", Electronic Journal of Applied Statistical Analysis: Decision Support Systems and Services Evaluation, Vol. 5, No. 1, pp. 57-66, 2014.

[12] Q.A. Al-Radaideh and E. Al Nagi, "Using data mining techniques to build a classification model for predicting employees performance", International Journal of Advanced Computer Science and Applications, Vol. 3, No. 2, pp. 144-151, 2012.

[13] C.F. Chien and L.F. Chen, "Data mining to improve personnel selection and enhance human capital: A case study in high-technology industry. Expert Systems and Applications", Expert Systems with Applications, Vol. 34, No. 1, pp. 280-290, 2008.

[14] Z. Zhao and H. Liu, "Semi-supervised feature selection via spectral analysis", In: Proc. of the 2007 SIAM International Conference on Data Mining, pp. 641-646, 2007.

[15] L. A. Zadeh, "Fuzzy sets", Information and Control, Vol. 8, No. 3, pp. 338-353, 1965.

[16] JJ. Rada-Vilela, fuzzylite a fuzzy logic control library in $\mathrm{C}++$, The FuzzyLite Libraries for Fuzzy Logic Control, 2013 